\title{
Microcirculatory Effects of Selective Endothelin-A Receptor Antagonism in Testicular Torsion
}

\author{
Zoltán Bajory, * Renáta Varga, Ágnes Janovszky, László Pajor and Andrea Szabó \\ From the Departments of Urology (ZB, LP) and Oral and Maxillofacial Surgery (RV), Institute of Surgical Research (AJ, AS), \\ University of Szeged, Szeged, Hungary
}

Purpose: Testicular torsion without timely intervention causes incurable damage to the testis. We examined the causative role of microcirculatory injury in torsion induced testicular damage with particular regard to endothelin-A receptor activation.

Materials and Methods: The microcirculatory consequences of testicular torsion were assessed in the presence or absence of endothelin-A receptor antagonism in rats. Microcirculatory perfusion changes (red blood cell velocity and pulsatile flow pattern alterations) were examined by an orthogonal polarization spectral imaging technique. Microcirculatory inflammatory alterations were assessed by fluorescence intravital video microscopy after 60 -minute torsion followed by 240-minute reperfusion. As a specific endothelin-A receptor inhibitor, the antisense homology box derived peptide ETR-p1/fl was applied 10 minutes before reperfusion. Tissue accumulation of leukocytes was estimated by myeloperoxidase activity in tissue biopsies taken at the end of the 4-hour reperfusion period. In further experiments testicular weight as a marker of permanent damage was evaluated 45 days after torsion.

Results: The physiological pulsatile flow pattern ceased in the initial phase of reperfusion while leukocyte-endothelial interactions increased throughout the examined reperfusion period. Endothelin-A receptor antagonism caused earlier return of pulsatile flow and recovery of red blood cell velocity, and alleviated microcirculatory inflammatory reactions and atrophy.

Conclusions: Results suggest a pathophysiological role of endothelin-A receptor activation in the pathogenesis of testicular torsion. This effect is related to deterioration in testicular perfusion and activation of microcirculatory inflammatory reactions.

Key Words: testis; torsion, mechanical; receptor, endothelin A; reperfusion; microcirculation
DesPiTE reestablishment of blood flow testicular atrophy and aspermatogenesis are a common outcome of testicular torsion. ${ }^{1}$ Testicular injury pathology has been extensively investigated and various pharmacological approaches have been tested that target different levels of the pathomechanism, eg sildenafil citrate, dehydroepiandrosterone, propofol, morphine, quercetin, trapidil, erdosteine, dipyridamole, nonsteroid antiinflammatory drugs, antioxidants, etc. $^{2}$ In regard to the circulatory aspect examination methods usually reveal only partial recovery of blood flow at the microcirculatory level during the post-torsion period.

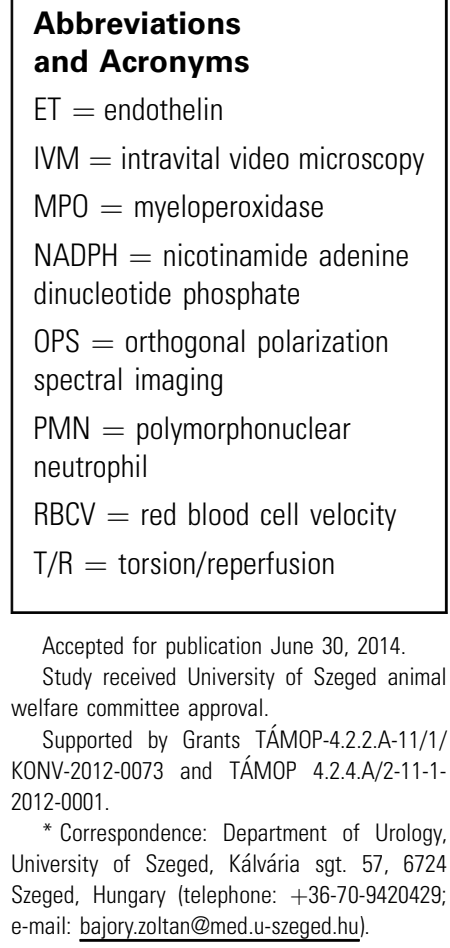

Accepted for publication June 30, 2014

Study received University of Szeged animal welfare committee approval.

Supported by Grants TÁMOP-4.2.2.A-11/1/ KONV-2012-0073 and TÁMOP 4.2.4.A/2-11-1. 2012-0001.

${ }^{*}$ Correspondence: Department of Urology, University of Szeged, Kálvária sgt. 57, 6724 Szeged, Hungary (telephone: $+36-70-9420429$; e-mail: bajory.zoltan@med.u-szeged.hu). 
Permanent loss of spermatogenesis was observed in a model of testicular torsion despite restoration of blood flow within 4 hours, as evidenced by a radioactive microsphere technique. ${ }^{1}$ In similar settings laser Doppler flowmetry indicated that flow was not sufficiently restored in the early stages but rather in the later phases of reperfusion. ${ }^{3}$ We similarly reported disturbed perfusion using in vivo microscopic methods with comparatively high spatial resolution, including fluorescence IVM and OPS. ${ }^{4}$ Another important microcirculatory phenomenon is PMN recruitment in subtunical venules. Their presence is closely linked to germ cell apoptosis. ${ }^{1}$

Reestablishing testicular microvascular perfusion and the balance between vasoconstrictor and vasodilator factors after torsion have a crucial impact on tissue survival. Some of these factors are derived from endothelium, including the vascular smooth muscle dilator nitric oxide and the vasoconstrictor ET-1, mainly via its ET-A receptors. Interestingly each vasoactive factor has a role in germ cell injury. ${ }^{5}$ Hypoxia is a major signal of ET-1 release. ${ }^{6}$ ET-1 is liberated from endothelium and also produced in nonvascular testicular tissues (seminiferous tubules, ${ }^{7}$ and Leydig and Sertoli cells $^{8}$ ). ET-1 induces oxidative stress by interfering with the NADPH oxidase system. In view of these findings the potential roles of ET-1 in the testis include regulation of blood flow via its direct effect on ET-A receptors or secondarily through NADPH oxidase derived superoxide production and modulation of spermatogenesis/germ cell apoptosis by similar free radical mediated mechanisms. ${ }^{5}$

Via calcium mobilization from intracellular and extracellular sources ET-A receptors promote vasoconstriction, smooth muscle cell proliferation and inflammation. ET-B receptors have opposing actions, causing vasodilation and increased sodium excretion, and inhibiting cell growth and inflammation. ${ }^{9}$ Several studies revealed that plasma ET-1 levels are increased, for example during myocardial ischemia/reperfusion injury, ${ }^{10}$ but alleviated by ET-A receptor antagonism. ${ }^{11}$ In the testis the density of ET-A receptors is considerably higher than that of ET-B receptors. ${ }^{12}$ Topical application of ET-1 gives rise to testicular vasoconstriction and leukocyte accumulation, which can be ameliorated by ET-A antagonism, ${ }^{8}$ but microvessels mostly show ET-B receptor positivity. ${ }^{7}$ Furthermore, nonselective ET receptor antagonism greatly ameliorates torsion induced testicular injury. ${ }^{5}$

A selective ET-A receptor antagonist, antisense homology box derived ETR-p1/fl peptide, ${ }^{13}$ also exerts positive effects in different types of ischemia/ reperfusion injury, of which some effects are related to microcirculatory improvement. It ameliorates postischemic mucosal injury and microcirculatory failure of the bowel. ${ }^{14}$ However, to our knowledge the microcirculatory effects of ET-A antagonism in the testis are unknown. We examined testicular microcirculatory reactions in response to testicular $\mathrm{T} / \mathrm{R}$ with particular interest in the role of ET-A receptor activation via the use of ETR-p1/fl peptide.

\section{MATERIALS AND METHODS}

Experiments were approved by the University of Szeged animal welfare committee and performed in accordance with National Institutes of Health Guidelines.

\section{Experimental Protocol}

A total of 51 male Sprague Dawley ${ }^{\circledR}$ rats weighing a mean $\pm \mathrm{SD} 250 \pm 10$ gm were randomly allotted into acute and chronic experimental series. In the acute series 31 rats were allotted into a further 6 groups. In the first 4 groups testicular microcirculation was examined by IVM or OPS 10 minutes after surgical exposure before experimental torsion, and at 15 and 30 minutes, and each hour of the 4-hour reperfusion period. In 2 of these 4 groups ETR-p1/fl peptide was administered intravenously as a single $0.25 \mathrm{mg} / \mathrm{kg}$ dose dissolved in $1 \mathrm{ml} / \mathrm{kg}$ saline, as based on a former study, ${ }^{15} 10$ minutes before reperfusion. Thus, 5 rats received T/R plus ETR-p1/fl-IVM and 5 received T/R plus ETR-p1/fl-OPS. Animals in the other 2 groups in this series served as controls. They received $1 \mathrm{ml} / \mathrm{kg}$ saline (T/R plus saline groups), and IVM and OPS recordings were made in 5 and 6 rats, respectively. Data were compared with those on 2 sham operated groups that received saline, including 5 rats in which IVM was done and 5 in which OPS was done. At the end of all experiments with OPS the affected and contralateral testes (16) were harvested to analyze MPO activity, which indicates tissue accumulation of PMNs.

In the chronic series a total of 20 rats were allotted to 3 experimental groups, including 8 rats with T/R plus ETR-p1/fl, 6 with T/R plus saline and 6 with sham operation plus saline. Intravenous injections of ETR-p1/f peptide or saline vehicle were administered in the penile veins. At 45 days the torsed testes together with the contralateral testes were harvested using pentobarbital anesthesia.

\section{Surgical Exposure}

In the acute series rats were anesthetized by intraperitoneal injection of sodium pentobarbital $(45 \mathrm{mg} / \mathrm{kg})$ and microcirculatory examinations were performed. After cannulating the trachea, the jugular vein and carotid artery were also cannulated to administer fluid and drugs, and measure arterial pressure using a device (Experimetria, Budapest, Hungary). The rat was placed supine on a heating pad. Ringer lactate was infused at a rate of $10 \mathrm{ml} / \mathrm{kg}$ per hour together with supplementary doses of pentobarbital $(5 \mathrm{mg} / \mathrm{kg})$ intravenously as necessary. Using a microsurgical approach a $2.0 \mathrm{~cm}$ incision was made in the scrotum, the internal spermatic fascia was excised and the testis was divided from the epididymis. The tunica albuginea and vessels remained intact. Clockwise 720-degree torsion was created and the testis was temporarily replaced in the scrotum, which was closed 
with a miniclip. Complete ischemia was verified by IVM in preliminary experiments. Torsion was reversed after 60 minutes.

In the 3 chronic groups testicular weight changes were determined 45 days after inducing experimental T/R. Short-term anesthesia was initiated by intraperitoneal injection of ketamine $(90 \mathrm{mg} / \mathrm{kg})$ and xylazine $(10 \mathrm{mg} / \mathrm{kg})$, and maintained by small empirical doses. The scrotum was disinfected with povidone iodide and the testis was prepared by the method described, followed by 720-degree torsion. At 60 minutes torsion was reversed, incisions were closed with sterile surgical threads (5-zero Vicryl®) and the rats were allowed to awaken.

\section{Testicular Microcirculatory Perfusion Changes}

Microcirculatory perfusion changes were assessed by the OPS technique using the Cytoscan ${ }^{\circledR}$. This technique uses epi-illumination with linearly polarized light to visualize structures containing hemoglobin without using fluorochrome. The left testis was positioned on a pedestal. Videomicroscopic images were recorded with a Panasonic AG-MD 830S-VHS video recorder (Matsushita, Tokyo, Japan). Microcirculatory parameters were quantitatively assessed off line by frame-to-frame analysis. Capillary RBCVs were measured in $\mu \mathrm{m}$ per second in 3 separate fields with at least 10 measurements per time point by a computer assisted image analysis system (IVM Pictron, Budapest, Hungary). Since normal testicular flow is characterized by vasomotion consisting of high and low flow periods, RBCV values of high flow periods were determined together with their relative duration.

\section{Testicular Neutrophil-Endothelial Cell Interactions}

The testis was positioned on a stage, superfused with 37C saline and visualized by IVM using an Axiotech Vario 100HD microscope, a $100 \mathrm{~W}$ HBO mercury lamp and an Acroplan $20 \times$ water immersion objective (Carl Zeiss, Jena, Germany). Fluorescein isothiocyanate labeled erythrocytes $(0.2 \mathrm{ml}$ intravenously) was used to stain red blood cells ${ }^{16}$ and rhodamine-6G $(0.2 \%$ in $0.1 \mathrm{ml}$ intravenously) (Sigma-Aldrich ${ }^{\circledR}$ ) was used to stain leukocytes. Recordings were made with a BC 12 charge-coupled device video camera (ATV-Horn, Aalen, Germany) attached to an S-VHS video recorder and a personal computer. Microcirculatory parameters were quantitatively evaluated off line by frame-to-frame analysis of videotaped images. Leukocyte-endothelial cell interactions were analyzed in at least 5 postcapillary venules per rat. Rolling leukocytes were defined as cells moving at a velocity of less than $40 \%$ of that of erythrocytes in the centerline of the microvessel that passed through within 30 seconds. Adherent leukocytes were defined as cells that did not move from the endothelial lining within 30 seconds. Since all freely flowing, rolling and adherent PMNs were clearly visible in this setup, values are shown as the percent per $20 \mu \mathrm{m}$ postcapillary venule segment.

\section{Measurement}

MPO activity. As a marker of tissue leukocyte infiltration, tissue MPO activity was measured in testicular biopsies according to a method modified from that of Kuebler et al. ${ }^{17}$ Briefly, tissue was homogenized with tris-HCl buffer (0.1 M, pH 7.4) containing $0.1 \mathrm{mM}$ polymethylsulfonyl fluoride to block tissue proteases and then centrifuged at $4 \mathrm{C}$ for 20 minutes. Sample MPO activity was measured at $450 \mathrm{~nm}$ using a UV-1601 spectrophotometer (Shimadzu, Kyoto, Japan). Data were normalized to protein content.

Testicular weight. Left testicular wet weight was measured 45 days after experimental torsion and compared to that of the contralateral testes.

\section{Statistical Analysis}

Statistical analysis was performed with SigmaStat ${ }^{\circledR}$ for Windows ${ }^{\circledR}$. Differences between groups were compared by 1-way ANOVA followed by the Holm-Sidak post hoc test. Values are shown as the mean \pm SEM with $\mathrm{p}<0.05$ considered significant.

\section{RESULTS}

\section{Testicular Microcirculatory Alterations}

Testicular microcirculation was characterized by a pulsatile flow pattern in all 5 rats in the sham operated, vehicle treated group during the entire examination period (see table). Thus, when a pulsatile flow pattern was present, RBCV during the high and low flow periods was determined

Time course of changes in capillary flow motion in sham operated rats and rats with T/R treated with ETR-pl/fI peptide or saline 10 minutes before reperfusion based on OPS data

\begin{tabular}{|c|c|c|c|c|c|c|c|}
\hline & Baseline & \multicolumn{6}{|c|}{ Reperfusion (mins) } \\
\hline \multicolumn{8}{|l|}{ Sham operated + saline: } \\
\hline Mean \pm SEM No. flow motion cycles $(1 / \mathrm{min})^{*}$ & $6.7 \pm 0.2$ & $6.6 \pm 0.6$ & $6.1 \pm 0.1$ & $6.9 \pm 0.9$ & $6.5 \pm 0.7$ & $6.5 \pm 0.4$ & $6.5 \pm 0.4$ \\
\hline Mean \pm SEM $\%$ high flow period relative duration & $75.5 \pm 2.0$ & $82.1 \pm 3.3$ & $81.5 \pm 2.7$ & $82.2 \pm 2.8$ & $78.8 \pm 2.3$ & $75.8 \pm 2.3$ & $79.7 \pm 2.6$ \\
\hline \multicolumn{8}{|l|}{$\mathrm{T} / \mathrm{R}+$ saline: } \\
\hline Flow motion incidence (No. rats) & 5 & 3 & 2 & 2 & 2 & 3 & 2 \\
\hline \multicolumn{8}{|l|}{ T/R + ETR-pl/fl: } \\
\hline Flow motion incidence (№. rats) & 5 & 3 & 3 & 4 & 4 & 3 & 4 \\
\hline Mean \pm SEM No. flow motion cycles $(1 / \mathrm{min})^{*}$ & $6.2 \pm 0.1$ & $9.3 \pm 0.4$ & $9.2 \pm 0.5$ & $10.6 \pm 0.6$ & $7.3 \pm 0.6$ & $7.4 \pm 0.7$ & $9.0 \pm 0.3$ \\
\hline Mean \pm SEM $\%$ high flow period relative duration & $76.2 \pm 2.1$ & $65.5 \pm 4.2$ & $68.4 \pm 5.6$ & $72.5 \pm 6.6$ & $63.8 \pm 2.9$ & $78.9 \pm 6.2$ & $77.4 \pm 3.9$ \\
\hline
\end{tabular}

* If present. 


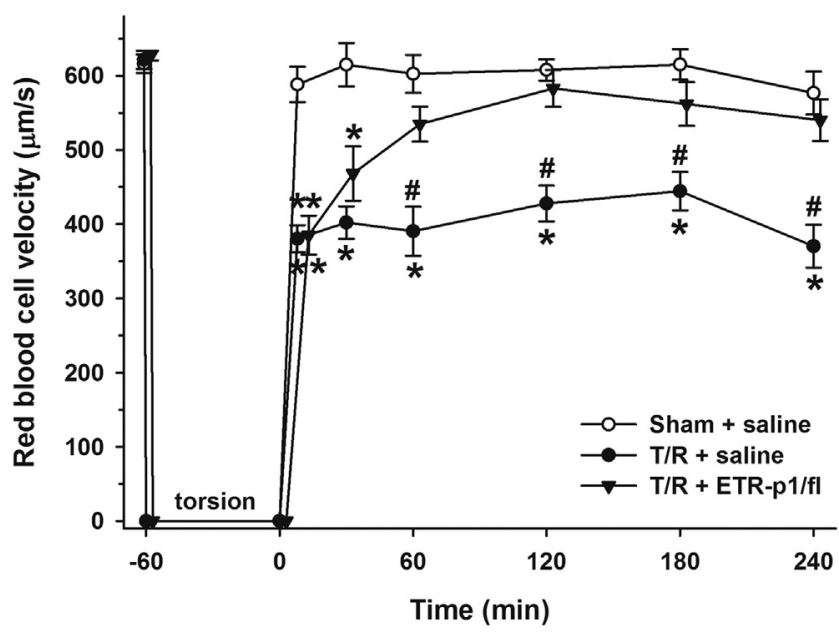

Figure 1. Mean \pm SEM RBCV in testicular microcirculation capillaries in sham operated, and T/R and T/R plus ETR-p1/fl treated rats. Single asterisk indicates $p<0.05$ vs sham operated plus saline. Pound sign indicates $p<0.05$ vs T/R plus ETR-p1/fl.

separately together with the corresponding duration in each group. In the sham operated group microvascular perfusion cycling with respect to RBCV shown during the high flow periods of flow motion (fig. 1), the number of corresponding cycles and the relative duration of the high flow periods were grossly unchanged throughout the observation period (table 1). However, in response to $T / R$ this flow pattern disappeared in many rats (it was present in 2 to 3 of 5) and continuous perfusion was noted. Therefore, RBCV at randomly chosen time points during the continuous flow pattern and RBCV measured during high flow periods were considered. This method allowed us to compare RBCV irrespective of the flow pattern in the $2 \mathrm{~T} / \mathrm{R}$ challenged groups.

This calculation revealed that $\mathrm{T} / \mathrm{R}$ significantly decreased RBCV in the saline treated group while recovery toward baseline was seen in the ETR-p1/fl treated group (fig. 1). The incidence of a pulsatile flow pattern was also considerably higher in the latter group (see table). The relative duration of high flow periods appeared to be greater in response to ET-A antagonism, although experimental numbers did not allow for statistical comparison.

\section{Neutrophil-Endothelial Interaction and MPO Activity Changes}

$T / R$ resulted in a particularly high increase in the number of rolling leukocytes in subtunical venules in saline treated rats (fig. 2, A). Due to the higher data dispersion there was merely a similar tendency toward leukocyte adherence (fig. 2, B). ETR-p1/fl peptide caused marker amelioration of each parameter (fig. 2), which was statistically significant throughout the reperfusion period for rolling leukocytes. Similar changes were seen in MPO values (fig. 3).

\section{Testicular Atrophy}

Testicular weight changes were evaluated 45 days after the T/R challenge. Sham operation did not influence testicular weight but $T / R$ resulted in a significant decrease in saline treated rats. These changes were ameliorated by administering ETR-p1/fl peptide (fig. 4).

\section{DISCUSSION}

In the current study selective ET-A antagonism resulted in significant amelioration of the microcirculatory inflammatory reactions caused by experimental $\mathrm{T} / \mathrm{R}$. These changes manifested in improvements in microvascular perfusion, neutrophilendothelial cell interactions and accompanying long-term testicular damage.

Restoring testicular blood flow is critical to preserve spermatogenesis. Even moderately acute
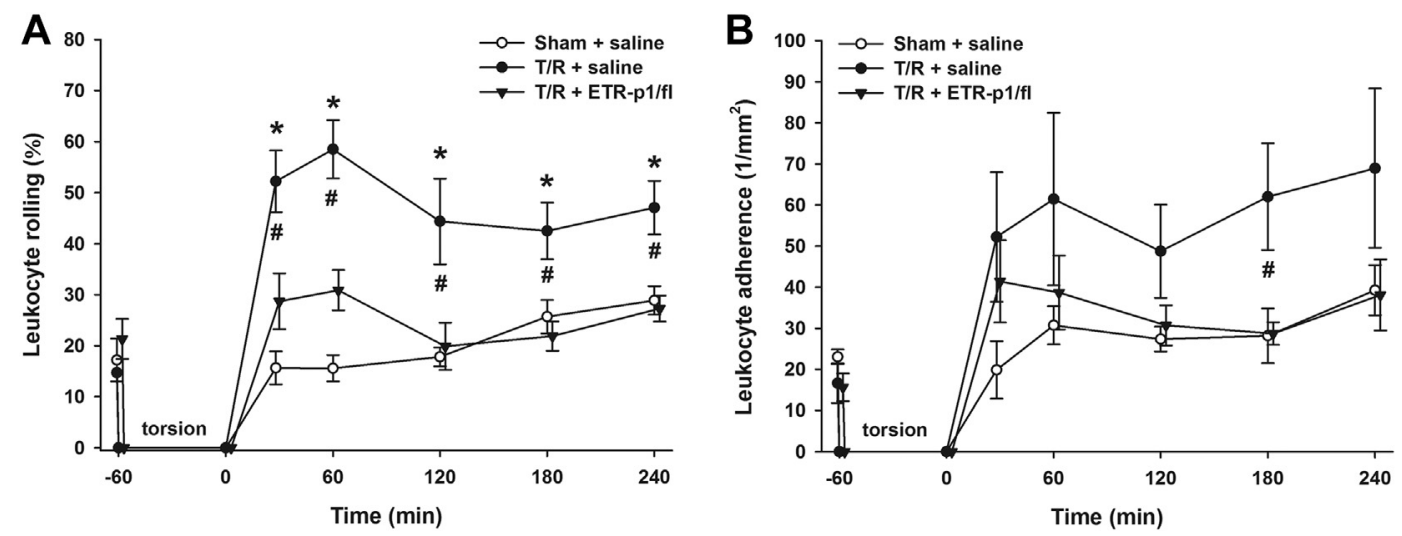

Figure 2. Number of rolling $(A)$ and adherent $(B)$ leukocytes in testicular subtunical venules in sham operated, and T/R and T/R plus ETR-p1/fl treated rats. Single asterisk indicates $p<0.05$ vs sham operated plus saline. Pound sign indicates $p<0.05$ vs T/R plus saline. 


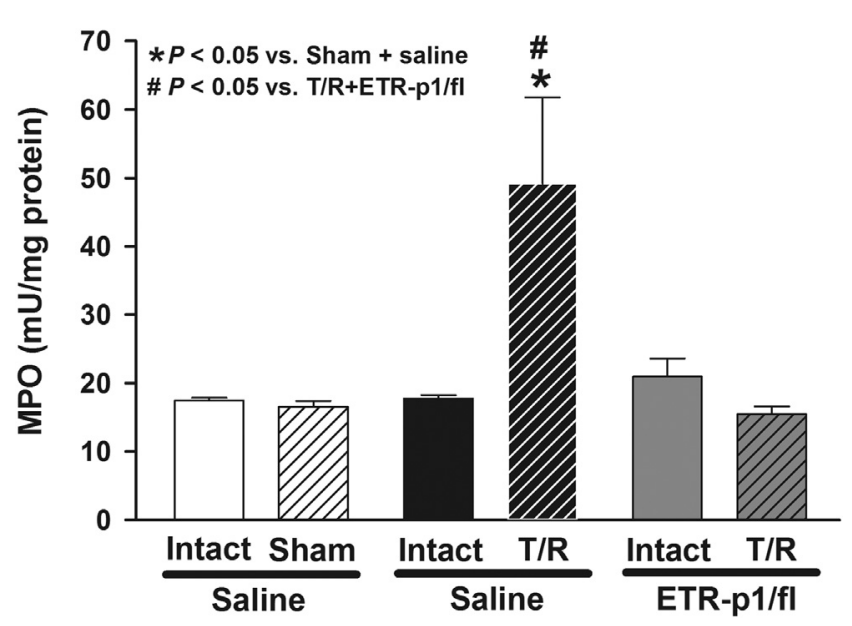

Figure 3. Mean \pm SEM testicular MPO activity after sham operation, T/R plus saline and ETR-p1/fl peptide treatment, and on contralateral side (Intact).

decreases in nutritive blood flow affect testicular morphology. The extent of flow reduction during the ischemic phase depends on the methodology applied, particularly in the clinical setting. These changes can be identified only with major limitations by perfusion CT, ${ }^{18}$ color Doppler ultrasound ${ }^{19}$ or perfusion scintigraphy. ${ }^{20}$ Therefore, the overall clinical diagnosis is based on clinical signs and physical examination.

During the reperfusion phase only partial testicular flow recovery is usually evidenced. Methods such as laser Doppler flowmetry used in experimental settings provide more detailed information on postischemic perfusion characteristics, indicating an approximately $70 \%$ return of baseline flow and the cessation of flow motion in the early

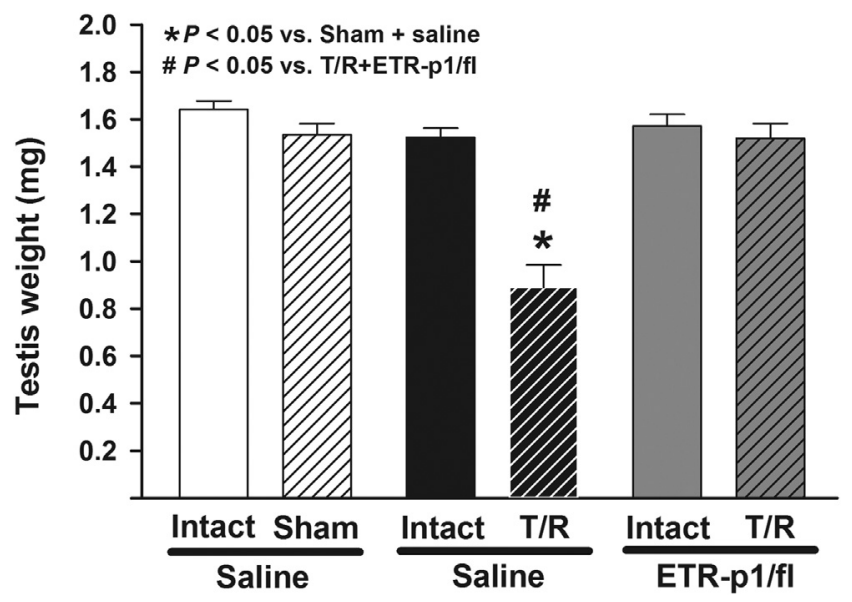

Figure 4. Mean \pm SEM testicular weight 45 days after sham operation, T/R plus saline and ETR-p1/fl peptide treatment, and on contralateral side (Intact). reperfusion stage. ${ }^{21}$ Marked perfusion and oxygenation deficits can also be discerned in the contralateral testis. Deterioration in postischemic microvascular flow and loss of its temporal variability were also revealed by the methodology that we applied in the current study. The lack of physiological flow motion and the concomitant cycling of tissue oxygen tension appear to be characteristic consequences of $T / R$, which showed only partial recovery by 24 hours and almost complete restoration at 7 days. Normal flow motion does not reappear after 1-hour testicular torsion even in the presence of normalized flow values. ${ }^{22}$ Improvements in microvascular perfusion achieved by any intervention should be based on simultaneous restoration of these parameters.

Although to our knowledge the underlying mechanisms of physiological testicular vasomotion and flow motion are not yet known, these reactions are implicated in the mediation of normal local transvascular fluid exchange ${ }^{23}$ and most probably signs of intact/restored testicular vasoregulation. Furthermore, vasomotion is influenced by hormonal factors ${ }^{24}$ and scrotal temperature. ${ }^{25}$ Flow motion is a physiological characteristic of testicular circulation but the phenomenon is considered a sign of impaired microcirculation in other organs. ${ }^{15}$ OPS, which allows for simultaneous detection of arteriolar vasomotion, flow motion and capillary flow, demonstrates that sinusoid changes in arteriolar diameter (vasomotion) are accompanied by almost square wave changes in microvascular flow (flow motion). In the testis Kolettis et al reported vasomotion alterations in the microvasculature of the seminiferous tubules and a simultaneous flow reduction in the supplying artery in response to contralateral $\mathrm{T} / \mathrm{R} .{ }^{21}$ In the current study RBCV in individual subcapsular capillaries was determined by OPS. Data support laser Doppler flowmetry findings in regard to the disappearance of capillary flow motion during the early post-torsion phase. Data analysis also revealed decreased RBCV in continuous flow situations and occasional high flow periods during reperfusion. Notably ET-A receptor antagonism with ETR-p1/fl peptide caused partial recovery of flow motion and also reversed RBCV decreases during recurring high flow periods.

Our study provides no direct evidence of the exact mode of action of ET-A antagonism on postischemic testicular microcirculation. However, it provides indirect confirmation of ischemia/reperfusion induced ET release and ET-A receptor activation. The balance between vasoactive mediators is shifted toward vasoconstriction because the capillary RBCV data indicate marked deterioration. Since capillaries lack the smooth muscle component, this reaction can be derived from vasoconstriction of 
upstream arterioles. ET-A antagonism not only ameliorated postischemic vasoconstriction but also seemed to restore arteriolar pacemaker activity, causing the reestablishment of vasomotion in most rats.

It is interesting that the testis and other vascular beds showed somewhat opposite reactions to hypoxia-reoxygenation injury. In an earlier study we noted activation of capillary flow motion in intestinal villi during hemorrhagic shock ${ }^{15}$ but the testis is characterized by an opposite reaction that is marked by the disappearance of physiological capillary flow motion. Furthermore, the positive effect of ETR-p1/fl treatment was seen in improvements in microvascular flow in each case but with different effects on flow motion. Nonetheless, the ET-A antagonist applied appeared to restore the original physiological characteristics of temporal flow variability.

A potential explanation of these reactions linked to vasospasm is the effect of ET-1 on NADPH oxidase dependent $\mathrm{O}_{2}{ }^{-}$production with resulting impairment of endothelium dependent vasodilatation. ${ }^{26}$ Activation of this pathway is implicated in diabetic testopathy but to our knowledge its role in $T / R$ induced testicular injury has not yet been defined. Nonselective ET receptor antagonism ameliorates testicular postischemic free radical production, apoptosis and tissue injury but the relative importance of tissue derived NADPH oxidase has not been clarified.

PMN leukocytes are another potential source of an oxidative stress induced increase in DNA damage and germ cell apoptosis. Margination and diapedesis of leukocytes occur within 4 hours after torsion repair. ${ }^{27}$ Activated extravasated neutrophils secrete important components of tissue injury such as growth factors, chemokines and cytokines, and they complement components such as proteases, nitric oxide, reactive oxygen metabolites and peroxynitrite at inflammatory sites. PMNs contribute to germ cell DNA damage and the initiation of mitochondrial apoptotic pathways. ${ }^{28}$ Tissue accumulation of PMNs is facilitated by proinflammatory cytokine induced increases in adhesion molecule expression while monoclonal antibodies against P-selectin ${ }^{29}$ and E-selectin ${ }^{30}$ dramatically decrease tissue invasion and apoptosis. In our study IVM demonstrated significant increases in leukocyteendothelial cell interactions in subtunical venules, followed by tissue accumulation as evidenced by MPO activity measurements. Notably the ETR-p1/fl peptide exerted a positive effect on these reactions.

This study has certain limitations because important indexes of tissue injury could not be examined, eg oxidative stress parameters, spermatocyte apoptosis or changes in endocrine function, fertility or testicular morphology. The relative contributions of temporal microvascular flow variability, perfusion deficit and PMN mediated pathways to overall testicular injury could not be clarified. In conclusion, it appears reasonable to assume that the beneficial effects of ET-A antagonism on PMN accumulation, acting in concert with those on the preservation/restoration of tissue perfusion, are involved in overall prevention of the testicular atrophy that was otherwise seen 45 days after the $\mathrm{T} / \mathrm{R}$ challenge.

\section{ACKNOWLEDGMENTS}

Prof. Hidechika Okada, Department of Immunology, Nagoya City University, Nagoya, Japan, provided ETR-p1/fl peptide.

\section{REFERENCES}

1. Turner TT and Brown KJ: Spermatic cord torsion: loss of spermatogenesis despite return of blood flow. Biol Reprod 1993; 49: 401.

2. DaJusta DG, Granberg CF, Villanueva $C$ et al: Contemporary review of testicular torsion: new concepts, emerging technologies and potential therapeutics. J Pediatr Urol 2013; 9: 723.

3. Lysiak JJ, Nguyen QA and Turner TT: Fluctuations in rat testicular interstitial oxygen tensions are linked to testicular vasomotion: persistence after repair of torsion. Biol Reprod 2000; 63: 1383.

4. Bajory Z, Szabó A, Deák G et al: Orthogonal polarization spectral imaging: a novel tool for examination of microcirculatory changes in the testis. J Androl 2012; 33: 499
5. Turkili B, Kurcer Z, Dengiz GO et al: Role of angiotensin and endothelin in testicular ischemia reperfusion injury. Int $\mathrm{J}$ Urol 2012; 19: 257.

6. Rakugi H, Tabuchi Y, Nakamaru M et al: Evidence for endothelin-1 release from resistance vessels of rats in response to hypoxia. Biochem Biophys Res Commun 1990; 169: 973.

7. Ergün $S$, Harneit $S$, Paust HJ et al: Endothelin and endothelin receptors $A$ and $B$ in the human testis. Anat Embryol (Berl) 1999; 199: 207.

8. Collin O, Damber JE and Bergh A: Effects of endothelin-1 on the rat testicular vasculature. J Androl 1996; 17: 360

9. Schneider MP, Boesen El and Pollock DM Contrasting actions of endothelin ET(A) and
ET(B) receptors in cardiovascular disease. Annu Rev Pharmacol Toxicol 2007; 47: 731.

10. Han B, Ghanim D, Peleg A et al: Loss of systemic endothelial function post-PCI. Acute Card Care 2008; 10: 79

11. Goyal SN, Bharti S, Arora $S$ et al: Endothelin receptor antagonist B0-123 ameliorates myocardial ischemic-reperfusion injury in rats: a hemodynamic, biochemical, histopathological and electron microscopic evidence. Biomed Pharmacother 2010; 64: 639.

12. Maggi $M$, Barni T, Orlando $C$ et al: Endothelin-1 and its receptors in human testis. J Androl 1995; 16: 213.

13. Baranyi L, Campbell W, Ohshima K et al: The antisense homology box: a new motif within 
proteins that encodes biologically active peptides. Nat Med 1995; 1: 894.

14. Wolfárd A, Szalay L, Kaszaki J et al: Dynamic in vivo observation of villus microcirculation during small bowel autotransplantation: effects of endothelin-A receptor inhibition. Transplantation 2002; 73: 1511.

15. Szabó A, Suki B, Csonka E et al: Flow motion in the intestinal villi during hemorrhagic shock: a new method to characterize the microcirculatory changes. Shock 2004; 21: 320.

16. Ruh J, Ryschich E, Secchi A et al: Measurement of blood flow in the main arteriole of the villi in rat small intestine with FITC-labeled erythrocytes. Microvasc Res 1998; 56: 62.

17. Kuebler WM, Abels C, Schuerer $L$ et al: Measurement of neutrophil content in brain and lung tissue by a modified myeloperoxidase assay. Int J Microcirc Clin Exp 1996; 16: 89.

18. Farhat WA: Perfusion CT evaluation in experimentally induced testicular torsion: proceed with caution. Can Urol Assoc J 2009; 3: 387.
19. Pepe P, Panella P, Pennisi $M$ et al: Does color Doppler sonography improve the clinical assessment of patients with acute scrotum? Eur J Radiol 2006; 60: 120.

20. Gezici A, Ozturk H, Buyukbayram $H$ et al: Effects of gabexate mesilate on ischemia-reperfusioninduced testicular injury in rats. Pediatr Surg Int 2006; 22: 435

21. Kolettis PN, Stowe NT, Inman SR et al: Acute spermatic cord torsion alters the microcirculation of the contralateral testis. J Urol 1996; 155: 350.

22. Becker EJ, Prillaman HM and Turner TT: Microvascular blood flow is altered after repair of testicular torsion in the rat. J Urol 1997; 157: 1493.

23. Bergh A and Damber JE: Vascular controls in testicular physiology. In: Molecular Biology of the Male Reproductive System. Edited by DM de Kretser. New York: Academic Press 1993; p 439.

24. Collin 0, Bergh A, Damber JE et al: Control of testicular vasomotion by testosterone and tubular factors in rats. J Reprod Fertil 1993; 97: 115 .
25. Setchell BP, Bergh A, Widmark A et al: Effect of testicular temperature on vasomotion and blood flow. Int J Androl 1995; 18: 120.

26. Duerrschmidt N, Wippich N, Goettsch W et al: Endothelin-1 induces NAD(P)H oxidase in human endothelial cells. Biochem Biophys Res Commun 2000; 269: 713.

27. Turner TT, Tung KSK, Tomomasa $\mathrm{H}$ et al: Acute testicular ischemia results in germ cell-specific apoptosis in the rat. Biol Reprod 1997; 57: 1267.

28. Lysiak JJ, Turner SD and Turner TT: Molecular pathway of germ cell apoptosis following ischemia/reperfusion of the rat testis. Biol Reprod 2000; 63: 1465

29. Celebi M and Paul AG: Blockade of p-selectin reduces neutrophil infiltration into the murine testis after ischemia-reperfusion-injury. Dtsch Tierarztl Wochenschr 2008; 115: 457.

30. Celebi M and Paul AG: Blocking E-selectin inhibits ischaemia-reperfusion-induced neutrophi recruitment to the murine testis. Andrologia 2008; 40: 235 . 